\section{Beschreibung der Versuche}

$\delta$-Aminolävulinsäure wird nach NEUBERger und ScotT $^{3}$ dargestellt. Die Fermentationen werden, wie bereits früher beschrieben ${ }^{1,4}$, unter Verwendung von Cornsteep oder eines synthetischen Nährmediums durchgeführt.

Gewinnung von Porphobilinogen. 80 g gefriergetrocknete Zellen (entsprechend $270 \mathrm{~g}$ Bakterienfeuchtmasse) werden in 21 Tris/HCl-Puffer $(\mathrm{pH} \mathrm{8,2,0,05 \textrm {M }}$ an Tris) suspendiert und während $30 \mathrm{Min}$. bei $70^{\circ} \mathrm{C}$ unter Rühren vorinkubiert. Anschließend wird abzentrifugiert und die Zellmasse erneut in der gleichen Menge Tris/HCl-Puffer gleicher Zusammensetzung suspendiert. Diese Suspension wird mit einer Lösung von $1,6 \mathrm{~g} \delta$-Aminolävulinsäure-hydrochlorid in $100 \mathrm{ml} \mathrm{H}_{2} \mathrm{O}$, deren $\mathrm{pH}$ mit Tris-Lösung auf 8,2 eingestellt wurde, versetzt und in einem 4-l-Rundkolben, der mit einem Wattestopfen verschlossen wird, unter Rühren (Magnetrührer) 20 Stunden bei $30{ }^{\circ} \mathrm{C}$ inkubiert. Dann wird von der Zellmasse abzentrifugiert und der $\mathrm{pH}$ der Lösung mit verdünnter $\mathrm{HCl}$ auf 7,2 eingestellt. Anschließend wird die Lösung auf eine kurze Säule $(5 \mathrm{~cm}$ Länge, 4,5 cm Durchmesser) aus DEAE-Cellulose (Fa. Merck, Darmstadt) gegeben, deren Chlorid-Form mit Tris-HClPuffer ( $\mathrm{pH} 7,2,0,05 \mathrm{~m}$ an Tris) so lange gewaschen worden war, bis der Durchlauf ein $\mathrm{pH}$ von 7,2 hatte. Es wird so lange mit diesem Puffer eluiert, bis kein Porphobilinogen mehr von der Säule kommt. Der $\mathrm{pH}$

1 G. Müller u. G. Bezold, Z. Naturforsch. 24 b, 47 [1969].

2 Literaturzusammenstellung: G. S. Marks: Heme and Chlorophyll, S. 129-130, Van Norstrand Company Ltd., London 1969.

3 A. Neuberger and J. J. Scott, J. chem. Soc. [London] 1954, 1820. des Eluats wird mit verdünnter $\mathrm{HCl}$ auf 4 eingestellt und das Porphobilinogen mit 20-proz. Quecksilber(II) acetat-Lösung gefällt. Der Niederschlag wird abzentrifugiert, mit $\mathrm{H}_{2} \mathrm{~S}$ zersetzt und vom $\mathrm{HgS}$ abzentrifugiert ${ }^{5}$. Dieses wird mehrere Male mit wenig Wasser extrahiert. Danach wird der $\mathrm{pH}$ der Lösung mit konz. Ammoniak auf 4 gestellt, wobei sich das Porphobilinogen abscheidet. Nach mehreren Stdn. im Kühlschrank werden die Kristalle abgesaugt, mit eiskalter verdünnter Essigsäure ( $\mathrm{pH} \mathrm{4)}$ und anschließend mit wenig kaltem Aceton gewaschen. Das so gewonnene Produkt ist in den meisten Fällen genügend rein.

Falls eine Umkristallisation notwendig ist, wird das Rohprodukt in möglichst wenig 0,5 N Ammoniak gelöst, eventuell filtriert, und bis zur Aufhellung etwas DEAE-Cellulose $\left(\mathrm{OH}^{\odot}\right.$-Form $)$ eingerührt. Nachdem vom Austauscher abfiltriert worden war, stellt man zur Fällung des Porphobilinogen den $\mathrm{pH}$ mit Eisessig vorsichtig auf 4 ein, läßt mehrere Stdn. im Kühlschrank stehen, saugt ab und trocknet im Exsiccator über $\mathrm{KOH}$. Ausbeute: $635 \mathrm{mg}$ ( $54 \%$ der Theorie).

Das Verfahren konnte ohne Schwierigkeiten auch mit größeren Mengen, z. B. in 8-l-Ansätzen, durchgeführt werden.

Der Deutschen Forschungsgemeinschaft und dem Verband der Chemischen Industrie bin ich für Personal- und Sachbeihilfen zu Dank verpflichtet.

4 G. Müller, W. Dieterle u. G. Siebke, Z. Naturforsch. 25 b, 307 [1970].

5 G. H. Cookson and C. Rimington, Biochem. J. 57, 476 [1954].

\section{A New Synthesis of Furo(2,3-b)quinoline}

\section{P. Shanmugam and P. Lakshminarayana}

Department of Organic Chemistry, University of Madras, Madras 25, India

(Z. Naturforsch. 27 b, 474-476 [1972] ; received November 16, 1971, revised December 24, 1971)

Furo(2,3-b)-quinoline (5) is the parent compound of many alkaloids ${ }^{1}$ of the Rutaceae. Of the two methods ${ }^{2,3}$ known for its synthesis, the one due to KING et al., involved in the final step, the catalytic dehydrogenation of the dihydrobase 8 with $\mathrm{Pd} / \mathrm{c}$. The base 8 is now obtainable ${ }^{4-6}$, through a shorter reaction sequence than the one employed by them ${ }^{2}$. But the dehydrogenation of $\mathbf{8}$ to $\mathbf{5}$ could not be effected inspite of repeated trials using varying strengths of the catalyst. The only published synthesis of $\mathbf{5}$ is that

Requests for reprints should be sent to Dr. P. Shanmugam, Reader in Organic Chemist University of Madras, A. C. College Campus, Madras 25 (India). of GRUNDON and McCorkindale who obtained it from a synthetic precursor to dictamnine. Apart from a number of steps involved, the yield at most of the stages leading to the precursor as well as its conversion to $\mathbf{5}$ is not appreciable. Hence a need to accomplish a neat and convenient synthesis of the title comcound was felt by us and this communication deals with such an objective realised through the use of 4-carboxy-3-vinyl-2-quinoline (1) ${ }^{7}$.
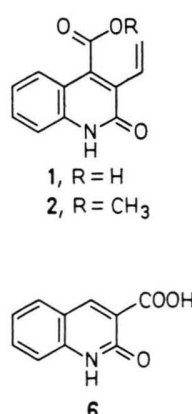

6
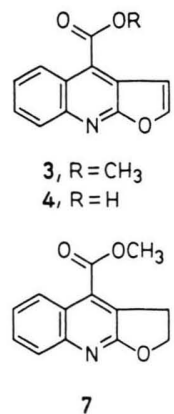<smiles></smiles>

5

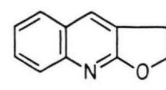

8 
Addition of bromine to its methyl ester 2 in chloroform and heating the resulting solution with triethylamine under reflux furnished the 4-carbomethoxy-furoquinoline (3). Similar conversion of a vinyl-quinolone to the corresponding furo $(2,3-b)$ quinoline has been reported ${ }^{8}$. The acid 4 derived from 3 by hydrolysis readily underwent decarboxylation with copper-bronze in boiling diphenyl ether to give the desired furoquinoline $\mathbf{5}$ in $65 \%$ yield.

3 and 5 underwent hydrogenation with $\mathrm{Pd} / \mathrm{c}$ and $\mathrm{H}_{2}$ to give $7^{2}$ and $8^{5}$ respectively. Permanganate oxidation $^{3}$ of 5 gave 2-quinolone-3-carboxylic acid, identical with the authentic sample ${ }^{9}$.

The N.M.R. spectra of the bases 3, 5, 7 and 8 are in accord with the structures assigned. The furan protons of 3 and 5 form an $\mathrm{AB}$ quartet $(\mathrm{J}=2.5 \mathrm{cps})$ as expected ${ }^{10}$. The $\mathrm{C}_{5}$ proton in 3 as well as in 7 appears at a lower field than the other aromatic protons, presumably due to deshielding by the carbomethoxy group.

\section{Experimental}

\section{4-Carbomethoxy-3-vinyl-2-quinolone (2)}

$3.3 \mathrm{~g} 1$ was refluxed with $8 \mathrm{ml}$ purified thionyl-chloride on a steam bath till it became homogeneous. After stripping off the excess reagent, the residue was taken in anhydrous chloroform and added to ice cold methanol $(20 \mathrm{ml})$. Refluxed for an hour, cooled and repeatedly washed with water and finally with sodium bicarbonate solution. After drying over anhydrous $\mathrm{MgSO}_{4}$, the solvent was removed and the residue weighing $3 \mathrm{~g}$ was straight-away used for the next reaction.

Analytically pure sample was however obtained by chromatography over alumina in benzene and elution with benzene-chloroform $(9: 1)$ evaporation and crystallisation from benzene gave rectangular plates. m.p. no sharp melting and it seems to undergo a thermal change above $150{ }^{\circ} \mathrm{C}$. (The ester with m.p. $149-150{ }^{\circ} \mathrm{C}$, erroneously indicated as 2 in a previous paper ${ }^{7}$ is the ester of the reduced acid, viz., 4-carboxy-3-ethyl-2 quinolone).

$$
\begin{aligned}
& \text { I.R. }(\mathrm{KBr}): 3436 \mathrm{~cm}^{-1}(-\mathrm{NH}-) ; 1733 \mathrm{~cm}^{-1} \\
& \left(\mathrm{COOCH}_{3}\right) ; 1647 \mathrm{~cm}^{-1}(-\mathrm{NHCO}-) \text {. } \\
& \mathrm{C}_{13} \mathrm{H}_{11} \mathrm{NO}_{3}(229.2) \\
& \text { Calc. C 68.11 H 4.84 N 6.11, } \\
& \text { Found C } 67.92 \text { H } 4.65 \text { N } 6.0 \text {. }
\end{aligned}
$$

\section{4-Carbomethoxy furo(2,3-b) quinoline (3)}

The crude ester ( $3 \mathrm{~g}$ ) was dissolved in anhydrous chloroform and $2.5 \mathrm{~g}$ bromine (in $10 \mathrm{ml}$ chloroform) was added in drops at room temperature. After the addition it was mixed with triethylamine $(8 \mathrm{ml})$ and heated under reflux for 90 minutes. The residue that left behind after removal of the solvent and the unused amine, was chromatographed over alumina in benzene. Yield: 1.8 g. Crystallised from benzene-petrol m.p. $150-150^{\circ} \mathrm{C}$.

I.R. $\left(\mathrm{KBr}\right.$ ) : $1717 \mathrm{~cm}^{-1}$ (no band at the amide-carbonyl-region).
$\operatorname{NMR}\left(\mathrm{CDCl}_{3}\right): \delta 4.1\left(\mathrm{~s}, 3 \mathrm{H},-\mathrm{COOCH}_{3}\right) ; \delta 7.15(\mathrm{~d}$, $\left.1 \mathrm{H}, \mathrm{H}_{3}\right) ; \delta 7.88\left(\mathrm{~d}, 1 \mathrm{H}, \mathrm{H}_{2}\right) ; \delta 8.83(\mathrm{q}, 1 \mathrm{H}$, $\left.\mathrm{H}_{5}\right) ; \delta 7.5-8.3\left(\mathrm{~m}, 3 \mathrm{H}, \mathrm{H}_{6}, \mathrm{H}_{7}, \mathrm{H}_{8}\right)$.

UV EtOH: $245 ; 339(\log \varepsilon 4.5195 ; 4.1218) \lambda \max (\mathrm{m} \mu)$. $\mathrm{C}_{13} \mathrm{H}_{9} \mathrm{NO}_{3} \quad(227.2)$
Calc.
C 68.72
H 3.99
N 6.16,
Found
C 68.74
H 4.01
N 6.2.

(b) Hydrogenation of $\mathbf{3}$ in ethanol using $\mathrm{Pd} / \mathrm{c}(2 \%)$ and $\mathrm{H}_{2}(30 \mathrm{lb})$ gave 7 , m.p. $109-110^{\circ} \mathrm{C}$ in $70 \%$ yield identical with authentic sample ${ }^{7}$ in all respects (m.p. mixed m.p. I.R., U.V. and N.M.R.) .

$\operatorname{NMR}\left(\mathrm{CDCl}_{3}\right): \delta 3.45\left(\mathrm{t}, 2 \mathrm{H}, \mathrm{H}_{3}\right) \mathrm{J}=8 \mathrm{cps} ; \delta 4.6$ $\left(\mathrm{t}, 2 \mathrm{H}, \mathrm{H}_{2}\right) \mathrm{J}=8 \mathrm{cps} ; \delta 3.92\left(\mathrm{~s}, 3 \mathrm{H}, \mathrm{COOCH}_{3}\right)$; $7.1-7.85\left(\mathrm{~m}, 3 \mathrm{H}, \mathrm{H}_{6}, \mathrm{H}_{7}, \mathrm{H}_{8}\right) ; \delta 8.28(\mathrm{q}$, $\left.1 \mathrm{H}, \mathrm{H}_{5}\right)$.

$\mathrm{C}_{13} \mathrm{H}_{11} \mathrm{NO}_{3}(229.2)$

Calc. C 68.11 H 4.84 N 6.11,

Found C $68.01 \quad$ H 4.69 N 6.30 .

4-Carboxy furo(2,3-b) quinoline (4)

$1.5 \mathrm{~g} 3$ was refluxed with $6 \mathrm{~N} \mathrm{HCl}(25 \mathrm{ml})$ for two hours, filtered hot and the filtrate cooled and diluted with water. The white precipitate that separated was collected and crystallised from aq. ethanol. Yied $1.4 \mathrm{~g}$ (95\%). m.p. $276-277^{\circ} \mathrm{C}$.

$$
\begin{aligned}
& \text { I.R. }(\mathrm{KBr}): 1709 \mathrm{~cm}^{-1} ; 1607 \mathrm{~cm}^{-1} ; 1527 \mathrm{~cm}^{-1} \text {. } \\
& \begin{array}{llllll}
\mathrm{C}_{12} \mathrm{H}_{7} \mathrm{NO}_{3}(213.2) \\
\text { Calc. } & \text { C } 67.61 & \mathrm{H} & 3.31 & \mathrm{~N} & 6.57, \\
\text { Found } & \text { C } 67.58 & \mathrm{H} & 3.47 & \mathrm{~N} & 6.9 .
\end{array}
\end{aligned}
$$

Furo(2,3-b)quinoline (5)

$200 \mathrm{mg} 4$ was heated with copper bronze (fine powder) in $15 \mathrm{ml}$ diphenyl ether under reflux for 3 hours. Cooled and $20 \mathrm{ml}$ ether added and extracted with $4 \mathrm{~N} \mathrm{HCl}(10 \mathrm{ml})$. The acid extract was basified, extracted with ether and dried over anhydrous $\mathrm{K}_{2} \mathrm{CO}_{3}$. Removal of the solvent and crystallisation of the residue from $n$-hexane gave $\mathbf{5}$ in fine colourless needles (120 mg) m.p. $78.2-79.2^{\circ} \mathrm{C}$ (lit. ${ }^{3}$ m.p. $76-77^{\circ} \mathrm{C}$ ). I.R. (KBr) : $1620 \mathrm{~cm}^{-1} ; 1575 \mathrm{~cm}^{-1}$.

N.M.R. $\left(\mathrm{CDCl}_{3}\right): \delta 6.85\left(\mathrm{~d}, 1 \mathrm{H}, \mathrm{H}_{3}\right) ; \delta 7.8(\mathrm{~d}, 1 \mathrm{H}$, $\left.\mathrm{H}_{2}\right) ; \delta 8.33\left(\mathrm{~s}, 1 \mathrm{H}, \mathrm{H}_{4}\right) ; \delta 7-8(\mathrm{~m}, 4 \mathrm{H}, \mathrm{Ar})$.

U.V.EtOH: $235 ; 308 \quad(\log \varepsilon \quad 4.6222 ; 4.0729)$ $\lambda \max (\mathrm{m} \mu)$.

$\mathrm{C}_{11} \mathrm{H}_{7} \mathrm{NO}(169.2)$

$\begin{array}{lllll}\text { Calc. } & \text { C } 78.09 & \text { H } & 4.17 & \text { N } 8.28, \\ \text { Found } & \text { C } 77.92 & \text { H } 3.98 & \text { N } 8.10 .\end{array}$

(b) Hydrogenation of 5 in ethanol using $\mathrm{Pd} / \mathrm{c}$ (10\%) and $\mathrm{H}_{2}(20 \mathrm{lbs})$ gave 8 in $60 \%$ yield m.p. $121-122{ }^{\circ} \mathrm{C}$ (petrol) identical with authentic sample ${ }^{5}$ (m.p. mixe m.p. superimposable I.R.). The N.M.R. spectrum agrees with the published data ${ }^{11}$.

$$
\begin{aligned}
& \mathrm{C}_{11} \mathrm{H}_{9} \mathrm{NO} \text { (171.2) } \\
& \begin{array}{llll}
\text { Calc. } & \text { C } 77.17 & \text { H } 5.30 & \text { N } 8.18 \text {, } \\
\text { Found } & \text { C } 76.9 & \text { H } 5.10 & \text { N } 8.02 .
\end{array}
\end{aligned}
$$

We thank Dr. K. Nagarajan3, CIBA Research Centre for NMR spectra, Mr. R. BALASUBRAMANIAN for the analysis and Professor S. Swaminathan for his encouragement. One of us (P. L. N.) wishes to thank the U. G. C. for a Fellowship. 
1 H. T. Openshaw, Alkaloids 9, 226 [1967].

2 Unpublished work by King, LATham, and Patridge mentioned by Price, Fortschor, Chem. Org. Naturstoffe 13, 320 [1956].

3 M. F. Grundon and N. J. McCorkindale, J. chem. Soc. [London] 1957, 2177.

4 G. Kobayashi and Y. Kuwayama, J. pharmac. Soc. Japan 79, 125 [1959].

5 J. P. John and P. Shanmugam, Proc. Indian Acad. Sci. 51,161 [1961].

${ }^{6}$ H. Zimmer, F. Haupter, J. Rothe, W. E. J. Schrof, and R. WAlteR, Z. Naturforsch. 18 b, 165 [1963].

Zur Kondensation von 3-Acyl-4-hydroxycumarinen mit 0 -Aminophenylglyoxal-dimethylacetal

The Condensation of 3-Acyl-4-hydroxy-coumarins with $o$-Aminophenylglyoxal-dimethylacetal

Mladen Trkovnik und Blanka Bobarević

Laboratorium für Organische Chemie des Chemischen Institutes der Universität Sarajewo, Jugoslawien

$$
\text { und }
$$

THOMAS KAPpe

Institut für Organische Chemie der Universität Graz

(Z. Naturforsch. 27 b, 476-477 [1972]; eingegangen am 21. Januar 1972)

\section{Herrn Professor Dr. Erich Ziegler zum 60. Geburtstag gewidmet}

$o$-Aminophenylglyoxal-dimethylacetal (2), welches in einfacher Weise aus 4-Hydroxy-carbostyril zugänglich ist $^{1}$, ist eine wertvolle Ausgangsverbindung für die Synthese des Indigos ${ }^{1}$ sowie von Carbostyril- und Chi-
7 P. Lakshminarayana, P. Shanmugam, and K. K. Balasu. BRAMANYAN, Tetrahedron Letters [London] 57, 4947 [1970].

8 P. Shanmugam and P. Lakshminarayana, Tetrahedron Letters [London] 25, 2323 [1971].

9 P. Freil ander and C. F. Goering, Chem. Ber. 17, 459 [1884].

10 A. V. Robertson, Aust. J. Chem. 16, 451 [1963].

11 N. S. Narasimhan, M. V. Paradkar, and R. H. Arulkar, Tetrahedron [London] 27, 1354 [1971].

nolin-4-aldehyden ${ }^{2}$. Die letztgenannte Verbindungsklasse (als Dimethylacetale geschützt) entsteht durch Kondensation von 2 mit Carbonylverbindungen, die in a-Stellung eine Methyl- oder Methylengruppe besitzen, im Sinne einer Friedländer- ${ }^{3}$ bzw. Pfitzinger. Chinolinsynthese ${ }^{4}$.

Überträgt man dieses Verfahren auf die Kondensation von $\mathbf{2}$ mit 3-Acyl-4-hydroxy-cumarinen $(\mathbf{1} \mathbf{a}-\mathbf{j})$, so erhält man in 3-Stellung mit dem 2-Chinolyl-4-aldehydrest substituierte 4-Hydroxy-cumarine $3 \mathbf{a}-\mathbf{j}^{\mathbf{5}}$. Die Ketone $\mathbf{1} \mathbf{a}-\mathbf{j}$ können durch KLOSA-Ziegler-Acylierung ${ }^{6-8}$ aus 4 -Hydroxy-cumarin und den entsprechenden Carbonsäuren in Gegenwart von $\mathrm{POCl}_{3}$ gewonnen werden.

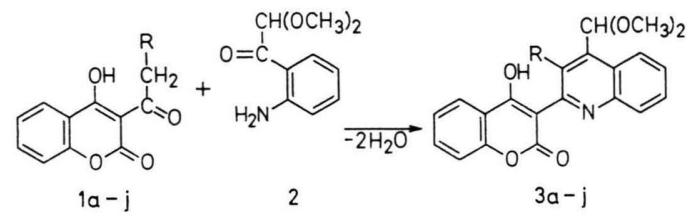

\begin{tabular}{|c|c|c|c|c|c|c|c|c|}
\hline & $\mathrm{R}$ & $\begin{array}{l}\text { Ausb. } \\
\% \text { d. Th. }\end{array}$ & $\begin{array}{l}\text { Schmp. } \\
{\left[{ }^{\circ} \mathrm{C}\right]}\end{array}$ & Summenformel & & $\begin{array}{l}\text { Analyse } \\
\mathrm{C}\end{array}$ & $\mathrm{H}$ & $\mathrm{N}$ \\
\hline $\mathbf{a}$ & $\mathrm{H}$ & 85 & $248-250$ & $\mathrm{C}_{21} \mathrm{H}_{17} \mathrm{NO}_{5}$ & Ber. & 69,40 & 4,73 & 3,86 \\
\hline & & & & & Gef. & 69,21 & 4,65 & 3,98 \\
\hline b & $\mathrm{CH}_{3}$ & 82 & $101-103$ & $\mathrm{C}_{22} \mathrm{H}_{19} \mathrm{NO}_{5}$ & $\begin{array}{l}\text { Ber. } \\
\text { Gef. }\end{array}$ & $\begin{array}{l}70,00 \\
70,24\end{array}$ & $\begin{array}{l}5,09 \\
5,36\end{array}$ & $\begin{array}{l}3,71 \\
3,62\end{array}$ \\
\hline e & $\mathrm{C}_{2} \mathrm{H}_{5}$ & 79 & $93-95$ & $\mathrm{C}_{23} \mathrm{H}_{21} \mathrm{NO}_{5}$ & Ber. & 70,55 & 5,43 & 3,58 \\
\hline & & & & & $\begin{array}{l}\text { Gef. } \\
\text { Ber. }\end{array}$ & $\begin{array}{l}70,68 \\
71,07\end{array}$ & $\begin{array}{l}5,24 \\
5,73\end{array}$ & $\begin{array}{l}3,79 \\
3,46\end{array}$ \\
\hline d & $n-\mathrm{C}_{3} \mathrm{H}_{7}$ & 75 & $88-90$ & $\mathrm{C}_{24} \mathrm{H}_{23} \mathrm{NO}_{5}$ & $\begin{array}{l}\text { Ber. } \\
\text { Gef. }\end{array}$ & $\begin{array}{l}71,07 \\
70,96\end{array}$ & $\begin{array}{l}5,73 \\
5,64\end{array}$ & $\begin{array}{l}3,46 \\
3,56\end{array}$ \\
\hline e & i- $\mathrm{C}_{3} \mathrm{H}_{7}$ & 76 & $91-93$ & $\mathrm{C}_{24} \mathrm{H}_{23} \mathrm{NO}_{5}$ & $\begin{array}{l}\text { Ber. } \\
\text { Gef. }\end{array}$ & $\begin{array}{l}71,07 \\
71,32\end{array}$ & $\begin{array}{l}5,73 \\
5,88\end{array}$ & $\begin{array}{l}3,46 \\
3,67\end{array}$ \\
\hline $\mathbf{f}$ & $n-\mathrm{C}_{4} \mathrm{H}_{9}$ & 78 & $87-88$ & $\mathrm{C}_{25} \mathrm{H}_{25} \mathrm{NO}_{5}$ & $\begin{array}{l}\text { Ber. } \\
\text { Gef. }\end{array}$ & $\begin{array}{l}71,59 \\
71,84\end{array}$ & $\begin{array}{l}6,02 \\
6,31\end{array}$ & $\begin{array}{l}3,34 \\
3,32\end{array}$ \\
\hline g & $n-\mathrm{C}_{5} \mathrm{H}_{11}$ & 74 & $83-85$ & $\mathrm{C}_{26} \mathrm{H}_{27} \mathrm{NO}_{5}$ & $\begin{array}{l}\text { Ber. } \\
\text { Gef. }\end{array}$ & $\begin{array}{l}72,04 \\
72,26\end{array}$ & $\begin{array}{l}6,28 \\
6,34\end{array}$ & $\begin{array}{l}3,23 \\
3,24\end{array}$ \\
\hline $\mathbf{h}$ & $n-\mathrm{C}_{6} \mathrm{H}_{13}$ & 75 & $77-79$ & $\mathrm{C}_{27} \mathrm{H}_{29} \mathrm{NO}_{5}$ & $\begin{array}{l}\text { Ber. } \\
\text { Gef. }\end{array}$ & $\begin{array}{l}72,48 \\
72,59\end{array}$ & $\begin{array}{l}6,54 \\
6,34\end{array}$ & $\begin{array}{l}3,13 \\
3,29\end{array}$ \\
\hline i & $n-\mathrm{C}_{7} \mathrm{H}_{15}$ & 77 & $72-74$ & $\mathrm{C}_{28} \mathrm{H}_{31} \mathrm{NO}_{5}$ & $\begin{array}{l}\text { Ber. } \\
\text { Gef. }\end{array}$ & $\begin{array}{l}72,86 \\
72,62\end{array}$ & $\begin{array}{l}6,78 \\
6,97\end{array}$ & $\begin{array}{l}3,03 \\
2,89\end{array}$ \\
\hline j & $n-\mathrm{C}_{8} \mathrm{H}_{17}$ & 72 & $81-83$ & $\mathrm{C}_{29} \mathrm{H}_{33} \mathrm{NO}_{5}$ & $\begin{array}{l}\text { Ber. } \\
\text { Gef. }\end{array}$ & $\begin{array}{l}73,22 \\
73,50\end{array}$ & $\begin{array}{l}7,01 \\
6,87\end{array}$ & $\begin{array}{l}2,95 \\
3,16\end{array}$ \\
\hline
\end{tabular}

Tab. 1. 2-(4-Hydroxy-cumarinyl-3)-4-dimethoxymethyl-chinoline $(3 \mathbf{a}-\mathbf{j})$.

Sonderdruckanforderungen an Doz. Dr. TH. KAPPE, Institut richstr. 28, Österreich. für Organische Chemie der Universität, $A-8010$ Graz, Hein- 